\title{
The low-mass stellar-mass functions of rich, compact clusters in the Large Magellanic Cloud ${ }^{\star}$
}

\author{
Q. Liu ${ }^{1,2}$, R. de Grijs ${ }^{3,1}$, L. C. Deng ${ }^{1}$, Y. Hu ${ }^{1,2}$, and S. F. Beaulieu ${ }^{4}$ \\ 1 National Astronomical Observatories, Chinese Academy of Sciences, Beijing 100012, PR China \\ e-mail: [liuq; licai; huyi]@bao.ac.cn \\ 2 Graduate University of the Chinese Academy of Sciences, Beijing 100049, PR China \\ 3 Department of Physics \& Astronomy, The University of Sheffield, Sheffield S3 7RH, UK \\ e-mail: R.deGrijs@sheffield.ac.uk \\ ${ }^{4}$ Département de Physique, de Génie Physique et d'Optique and Centre de Recherche en Astrophysique du Québec, \\ Université Laval, Québec, QC G1V 0A6, Canada
}

Received 14 April 2009 / Accepted 22 May 2009

\begin{abstract}
Context. The LMC is an ideal environment for studying stellar MFs, because it contains a large population of compact clusters at different evolutionary stages. We aim to obtain constraints on the initial MFs (IMFs) of our sample clusters on the basis of their present-day MFs, combined with our understanding of their dynamical and photometric evolution.

Aims. We use Hubble Space Telescope photometry for six rich, compact star clusters in the Large Magellanic Cloud (LMC), with ages ranging from 0.01 to $1.0 \mathrm{Gyr}$, to derive the clusters' stellar mass functions (MFs) at their half-mass radii.

Methods. We derived the clusters' present-day MFs below $1.0 M_{\odot}$ using deep observations with the Space Telescope Imaging Spectrograph and updated stellar population synthesis models.

Results. Since the relaxation timescales of low-mass stars are very long, dynamical evolution will not have affected the MFs below $1.0 M_{\odot}$ significantly, so that - within the uncertainties - the derived MFs are consistent with the solar-neighbourhood IMF, at least for the younger clusters. The IMF in the low-density, low-metallicity environment of the LMC disk is not significantly different from the IMF in the solar neighbourhood.
\end{abstract}

Key words. stars: low-mass, brown dwarfs - stars: luminosity function, mass function - stars: pre-main sequence Magellanic Clouds - galaxies: star clusters

\section{Introduction}

The shape of the stellar initial mass function (IMF) is a very important unresolved issue in modern astrophysics, because it plays a crucial role in many of the remaining "big questions". The IMF is usually assumed to be universal, and best approximated by either a power law (e.g., Kroupa 2001) or a lognormal distribution (Chabrier 2003; Andersen et al. 2008). Kroupa (2001) studied the Galactic-field IMF down to $0.01 M_{\odot}$ and derived a three-part power-law function. Chiosi et al. (2007) obtained a similar mass function down to $0.7 M_{\odot}$ based on their analysis of three clusters in the Small Magellanic Cloud (SMC), and concluded that the IMF in SMC clusters agrees with the "standard" Kroupa (2001) solar-neighbourhood IMF. Da Rio et al. (2009) has recently studied the stellar association LH 95 in the Large Magellanic Cloud (LMC), focusing on its pre-mainsequence (PMS) stars. Their results show that there are no significant differences between the IMFs of the entire LH 95 region and those of three individual subclusters, because they all follow a multiple power-law distribution. Andersen et al. (2008) studied the low-mass stellar mass distributions of seven starforming regions and concludes that the composite IMF is consistent with a lognormal distribution. Paresce et al. (2000) obtained a similar result after analysing the mass functions (MFs)

\footnotetext{
* Photometry Table is only available in electronic form at the CDS via anonymous ftp to cdsarc.u-strasbg.fr $(130.79 .128 .5)$ or via http://cdsweb.u-strasbg.fr/cgi-bin/qcat?J/A+A/503/469
}

of a dozen Galactic globular clusters (GCs) for stellar masses below $1.0 M_{\odot}$. Thus, the form of the universal IMF appears to be best approximated by both Kroupa (2001) broken power-law and lognormal distributions (Chabrier 2003; Andersen et al. 2008; Covey et al. 2008; Liu et al. 2009; Oliveira et al. 2009). To distinguish between either shape, one would need to probe down to the stellar/brown-dwarf transition region, which still poses a significant observational challenge, particularly in (even the nearest) extragalactic environments.

The evolution of the stellar MF is also more generally important because the IMFs of many clusters and galaxies cannot be observed directly. However, we can derive their IMFs based on the present-day MF (PDMF) if - at least - we understand its evolution in detail. Star clusters, both open clusters and GCs, provide ideal objects for tackling many astronomical problems, because all of their member stars have approximately the same age and metallicity, and are located roughly at the same distance. Although the GCs in the Milky Way are relatively nearby and their members can be observed easily, they are not well suited for studying the evolution of the MF, because Galactic GCs are all old (with ages $t \geq 10 \mathrm{Gyr}$ ). They can therefore only provide evolutionary information on long timescales. Galactic open clusters, on the other hand, are only effective tracers of MF evolution on short timescales, while they also tend to be affected quite significantly by low-number statistics. Ideally, therefore, we need rich massive clusters covering a wide age range to make significant progress on this important problem. This makes the LMC 
Table 1. Fundamental parameters of our LMC cluster sample.

\begin{tabular}{ccccccc}
\hline \hline Cluster & $\begin{array}{c}\log (\text { age })^{a} \\
{[\mathrm{yr}]}\end{array}$ & $\begin{array}{c}E(B-V)^{b} \\
(\mathrm{mag})\end{array}$ & $\begin{array}{c}(m-M)_{0}^{b} \\
(\mathrm{mag})\end{array}$ & $\log \left(M_{\mathrm{cl}} / M_{\odot}\right)^{c}$ & $\begin{array}{c}R_{\text {core }}^{c} \\
(\mathrm{pc})\end{array}$ & $\begin{array}{c}D_{\mathrm{LMC}}^{d} \\
\left({ }^{\circ}\right)\end{array}$ \\
\hline NGC 1805 & $7.65 \pm 0.05$ & 0.04 & 18.59 & $3.52 \pm 0.13$ & $1.33 \pm 0.06$ & $3.86-4.00$ \\
NGC 1818 & $7.65 \pm 0.05$ & 0.03 & 18.58 & $4.13_{-0.14}^{+0.15}$ & $2.45 \pm 0.09$ & $3.47-3.61$ \\
NGC 1831 & $8.75 \pm 0.05$ & 0.00 & 18.58 & $4.81 \pm 0.13$ & $4.44 \pm 0.14$ & $4.82-4.85$ \\
NGC 1868 & $9.00 \pm 0.05$ & 0.02 & 18.55 & $4.53 \pm 0.10$ & $1.62 \pm 0.05$ & $5.57-5.47$ \\
NGC 2209 & $9.10 \pm 0.05$ & 0.07 & 18.39 & $5.03_{-0.6}^{+0.36}$ & $5.43 \pm 0.33$ & $5.48-5.43$ \\
Hodge 14 & $9.30 \pm 0.05$ & 0.04 & 18.49 & $4.33_{-0.28}^{+0.34}$ & $1.80 \pm 0.14$ & $4.19-4.37$ \\
\hline
\end{tabular}

Notes: $D_{\mathrm{LMC}}$ is the distance from the LMC centre; the two values indicate $D_{\mathrm{LMC}}$ to the optical, geometrical centre (Bica et al. 1996) and the dynamical, rotation centre (Westerlund 1990), respectively. References: ${ }^{a}$ this paper (the age uncertainties are driven by the discreteness of the Padova isochrones); ${ }^{b}$ Castro et al. (2001); ${ }^{c}$ Mackey \& Gilmore (2003), ${ }^{d}$ Meurer et al. (1990).

an ideal laboratory, because it contains a large population of rich star clusters with masses similar to Galactic GCs and covering ages from 0.001 to 10 Gyr (e.g., Beaulieu et al. 1999; Elson et al. 1999). This implies that we can study the MF at almost all evolutionary stages using the rich, compact clusters in the LMC. In the past, it proved impossible to resolve individual stars in dense star clusters at the distance of the LMC ( $\sim 50 \mathrm{kpc})$, but the unprecedented, high spatial resolution of the Hubble Space Telescope (HST) facilitates such studies today.

In Sect. 2, we briefly describe our HST observations and give a basic overview of the data-reduction procedures (see detailed steps in Liu et al. 2009). We present and discuss our main results in Sects. 3 and 4, respectively, and provide a summary in Sect. 5.

\section{Observations and data reduction}

\subsection{Observations and previous work}

As part of HST programme GO-7307, we observed a carefully selected cluster sample in the LMC, including six compact clusters in three pairs (Pair I: NGC 1805 and NGC 1818, Pair II: NGC 1831 and NGC 1868, and Pair III: NGC 2209 and Hodge 14): see Table 1 for their fundamental parameters. Our six sample clusters have ages of $10^{7}-10^{9} \mathrm{yr}$, with the additional constraint that the two clusters in each pair have similar ages, metallicities, total mass, and distance from the LMC centre, yet different structural parameters (Beaulieu et al. 1999).

Much work has been done already, based on these highquality imaging observations (e.g., Elson et al. 1998, 1999; Johnson et al. 2001; Santiago et al. 2001; de Grijs et al. 2002a-c; Kerber et al. 2006; Liu et al. 2009). de Grijs et al. (2002a,b) obtained the MFs of the two youngest clusters in our sample for stellar masses above $1.0 M_{\odot}$, and concluded that they are largely similar to the Salpeter (1955) IMF. Liu et al. (2009) analysed the MF of NGC 1818 below $1.0 M_{\odot}$ and combined their low-mass MF with the higher-mass results of de Grijs et al. (2002b). They found that the IMF of NGC 1818 could be well approximated by both a Kroupa (2001)-type broken power-law function and a lognormal distribution.

Our high-quality imaging observations were obtained with both the Wide-Field and Planetary Camera-2 (WFPC2) and the Space Telescope Imaging Spectrograph (STIS). WFPC2 is composed of four chips (each containing $800 \times 800$ pixels), one planetary camera (PC) and three wide-field (WF) arrays (WF2, WF3, and WF4). The pixel size of the PC chip is 0.0455 arcsec (with a field of view of $\sim 34 \times 34 \operatorname{arcsec}^{2}$ ) and that of each WF chip is $0.097 \mathrm{arcsec}$ (with fields of view of $\sim 150 \times 150 \mathrm{arcsec}^{2}$ ). The STIS pixel size is 0.0507 arcsec and the corresponding field of view is about $28 \times 52 \operatorname{arcsec}^{2}$.
WFPC2 exposures through the $F 555 W$ and $F 814 W$ filters (roughly corresponding to the Johnson-Cousins $V$ and $I$ bands, respectively) were obtained with the PC chip centred on both the clusters' half-mass radii (with a total exposure time of $2500 \mathrm{~s}$ in both filters; see for more details Santiago et al. 2001; Grijs et al. 2002a). Deep STIS exposures in ACCUM imaging mode through the F $28 \times 50$ LP long-pass filter (central wavelength $\simeq 7230 \AA$ ) were also obtained, centred on the clusters' half-mass radii (with total exposure times of $2950 \mathrm{~s}$ for Pairs I and III, and 2890 s for Pair II; see Elson et al. 1999). To obtain "clean" MFs, we had to subtract the background stellar contribution. We therefore also obtained deep WFPC2 images from the HST Data Archive of the general LMC background through the $F 555 \mathrm{~W}$ and $F 814 \mathrm{~W}$ filters, with exposure times of 7800 and 5200 s, respectively (see Castro et al. 2001; Santiago et al. 2001; de Grijs et al. 2002a; Liu et al. 2009).

\subsection{Data reduction and photometry}

We used the same method for data reduction and photometry as in Liu et al. (2009). Aperture photometry ${ }^{1}$ was performed on our WFPC 2 and STIS images with the IRAF/APPHOT ${ }^{2}$ package, and 2-pixel apertures were adopted since this produced the smallest photometric errors and the tightest cluster main sequences.

We used the relations of Whitmore et al. (1999) to correct the resulting photometry for the time-dependent charge-transfer efficiency effects and IRAF/STSDAS ${ }^{3}$ to rectify the geometric distortions of the WFPC2 chips. We subsequently applied aperture corrections based on the model PSFs generated by TinyTim (Krist \& Hook 2001). Finally, we used the relations of Holtzman et al. (1995) to convert the aperture-corrected $F 555 W$ and $F 814 W$ magnitudes to the Johnson-Cousins $V$ and $I$ passbands. Figures 1 and 2 show, respectively, the resulting WFPC2-based colour-magnitude diagrams (CMDs) of all sample clusters and the spatial distributions of the stars in our LMC cluster fields as observed with both WFPC2 and STIS (dots and rectangular outlines, respectively).

\footnotetext{
1 See Liu et al. (2009) for a discussion of the pros and cons of using aperture photometry versus point-spread function fitting.

2 The Image Reduction and Analysis Facility (IRAF) is distributed by the National Optical Astronomy Observatories, which is operated by the Association of Universities for Research in Astronomy, Inc., under cooperative agreement with the US National Science Foundation.

${ }^{3}$ STSDAS, the Space Telescope Science Data Analysis System, contains tasks complementary to the existing IRAF tasks. Version 3.1 was adopted for the data reduction performed in this paper.
} 


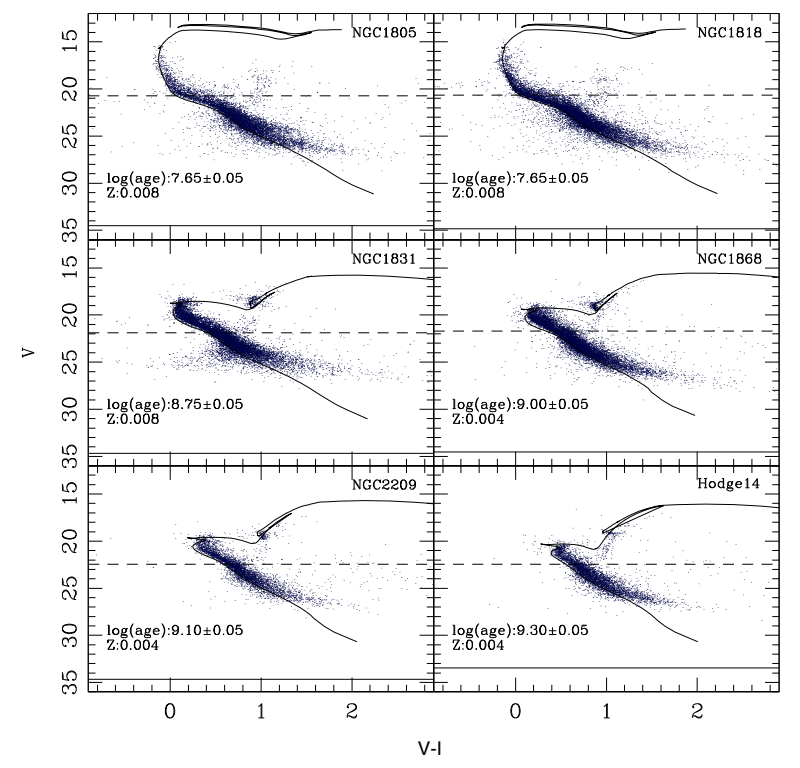

Fig. 1. Colour-magnitude diagrams of our LMC clusters and the bestfitting Padova isochrones (Girardi et al. 2000). The horizontal dashed and solid lines in each panel represent the upper and lower magnitude limits, respectively, of the parameter space covered by our STIS observations.
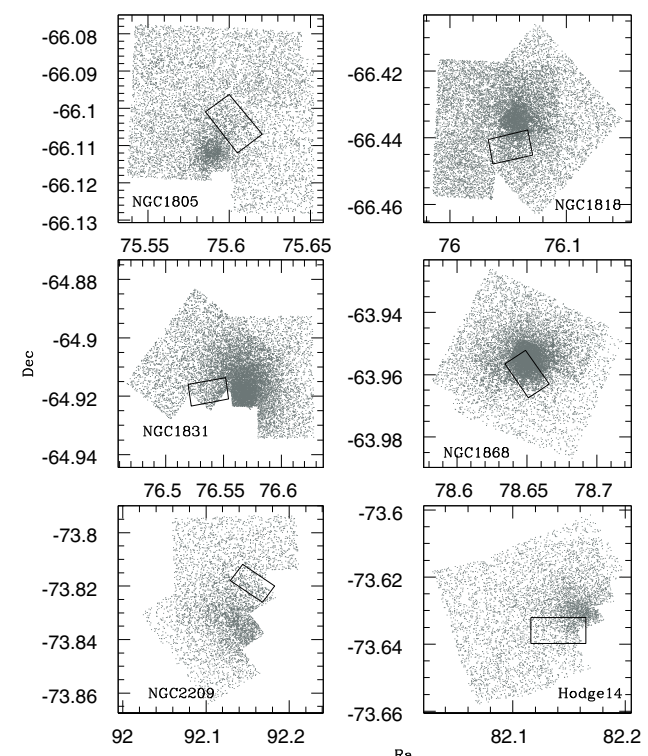

Fig. 2. Spatial distribution of the stars in our LMC clusters. The dots represent the stars detected in the WFPC2 observations, while the solid rectangular shapes indicate the areas covered by our STIS observations, which are used for further analysis in this paper.

\subsection{Completeness corrections and background subtraction}

One of the most difficult problems we faced in deriving clean MFs involved correcting for sampling incompleteness, which is usually a function of position in a cluster. We used the same method as in Liu et al. (2009), in essence a slightly modified version of the same approach as in de Grijs et al. (2002a), who computed these corrections in circular annuli around the cluster centres. We computed the completeness corrections for the entire STIS chip, because the STIS observations were centred on the low-density half-mass radius and the effects of sampling incompleteness are constant across our STIS field within the

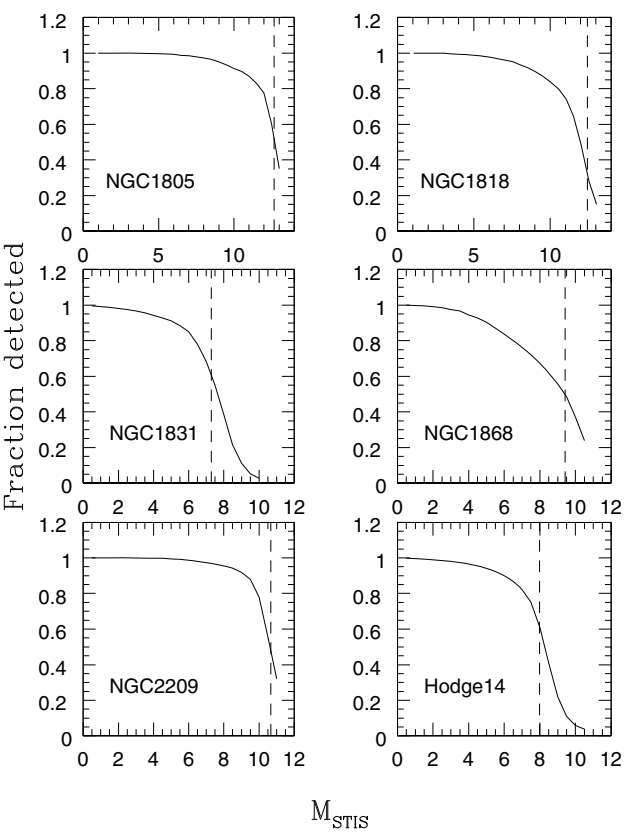

Fig. 3. Completeness ratios of all sample clusters. The dashed lines represent the $50 \%$ completeness limits for the individual clusters.

observational uncertainties (e.g., de Grijs et al. 2002a; Liu et al. 2009). We used the same method to compute the equivalent completeness corrections for the background field. We added an area-dependent number of artificial sources of Gaussian shape to each STIS exposure with input magnitudes between 16.0 and $30.0 \mathrm{mag}$, in steps of $0.5 \mathrm{mag}$. We then adopted the same photometric analysis method for the fields including both the "real" cluster (and background) stars and the artificial stars, to assess how many artificial stars could be detected after correction for blends and superpositions. For the analysis in this paper, we only consider magnitude ranges that are $\geq 50 \%$ complete.

To obtain a clean MF, we must subtract the contamination by the background field, because its stellar mass distribution is generally different from that of the clusters (cf. Castro et al. 2001). We do not have a background field in the STIS F28 $\times 50$ LP passband. Instead, we used a general WFPC2/F814W background-field observation. However, because our STIS observations are much deeper than the WFPC2 data, we could not directly subtract the full background effects from the observations. Gouliermis et al. (2006a) suggest that the stellar mass distribution in the LMC disk follows a broken power-law distribution. We therefore adopted a power law (with slope $\Gamma=1.87$, where the IMF, $\left.\xi\left(m_{*}\right) \propto m^{\Gamma}\right)$ to approximate and extrapolate the stellar mass distribution in the general LMC background down to $0.1 M_{\odot}$ (see Liu et al. 2009, their Fig. 5).

In Figs. 3 and 4 we show the photometric completeness fractions and the resulting luminosity functions (LFs) for all of our sample clusters, respectively. The LFs have been fully completeness-corrected and background-subtracted. We only include magnitude bins for which the observational completeness fractions are greater than $50 \%$.

\section{Analysis}

\subsection{Age, metallicity, and evolutionary models}

Santiago et al. (2001), de Grijs et al. (2002b,c), and Kerber et al. (2006) studied the MFs of our sample clusters above $1.0 M_{\odot}$, based on WFPC2 data. Liu et al. (2009) studied the MF of 


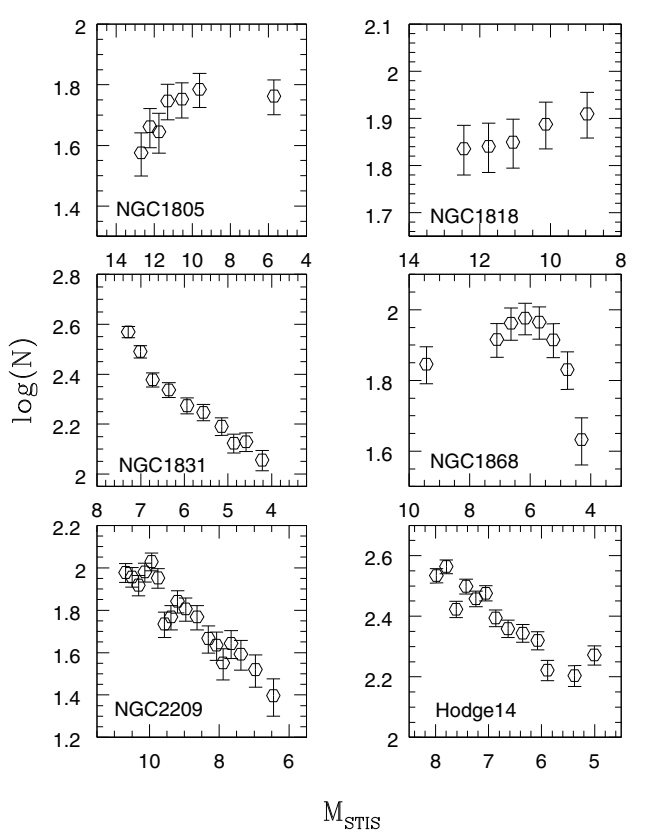

Fig. 4. Completeness-corrected, background-subtracted LFs of all sample clusters, for completeness fractions $\geq 50 \%$.

NGC 1818 below $1.0 M_{\odot}$ based on STIS data. Because the STIS data are much deeper than the WFPC2 measurements (e.g., de Grijs et al. 2002a), here we study the MFs of all six LMC clusters from HST programme GO-7307 based on the STIS observations.

The ages of our six sample clusters cover a wide range from $10^{7}-10^{9} \mathrm{yr}$. Most of the stars below $1.0 \mathrm{M}_{\odot}$ of the clusters in the youngest of pair, NGC 1805 and NGC 1818, are still on the PMS (Liu et al. 2009). The evolution of PMS stars is still uncertain (Baraffe et al. 1997, 1998). White et al. (1999) concluded that the models of Baraffe et al. $(1997,1998)$ resulted in the most consistent ages and masses, on the basis of a comparison of six PMS evolutionary model sets (Park et al. 2000). Because our STIS observations were obtained only in a single passband, we cannot derive the PMS ages and metallicities on the basis of CMD analysis. However, the cluster stars from our WFPC2 and STIS observations occupy a common locus on the CMD, so we can obtain the basic cluster parameters by adopting Padova isochrones (Girardi et al. 2000) to fit the faint end of the main sequence, and the Baraffe et al. (1997, 1998) models for the sequence of low-mass stars, i.e., by extrapolating the WFPC2 observations to fainter luminosities (see, for details, Liu et al. 2009).

The other sample clusters are old enough for all low-mass stars to have evolved onto the main sequence, so that we can get their ages and metallicities using Padova isochrones for mainsequence fitting. The best fits and the resulting basic cluster parameters are shown in Fig. 1. We used the Padova isochrones to fit the main-sequence ridge line in the CMD with different metallicities and ages for each cluster. Next, we compared the quality of all fits and adopted the best-fitting metallicity and age for the cluster of interest. The narrow spread of the main-sequence data points will cause a small uncertainty in the age determination for each cluster, which we characterise by using the discreteness of the Padova isochrones (in steps of 0.05 dex in age) as a proxy for the age uncertainties. In fact, for each cluster the nearest isochrones to the best-fitting model provide markedly worse fits to the main-sequence turn-off location, so that we are confident that our fits are robust.
In Liu et al. (2009) we added the $F 28 \times 50 L P$ filter to the Baraffe et al. (1998) model suite. These models cover a range of metallicities, enabling us to choose the most relevant massluminosity relation for conversion of the $F 28 \times 50 L P$ magnitudes to individual stellar masses.

A significant body of work exists in the literature to support our choice of metallicity for the individual clusters. For NGC 1805, Johnson et al. (2001) used HST CMDs to derive a mean $[\mathrm{Fe} / \mathrm{H}] \sim 0$ (solar metallicity), although Meliani et al. (1994) had argued previously that the cluster's most appropriate metallicity was $Z=0.008$ (where $Z_{\odot}=0.020$ ), i.e., the average metallicity of the young LMC field population. On this basis, we adopted $Z=0.008$ for this cluster, corresponding to $[\mathrm{Fe} / \mathrm{H}] \simeq-0.37$ (assuming a one-to-one correlation between metallicity and iron abundance). Using solar metallicity instead, we derive an age for NGC 1805 of $\log (t / \mathrm{yr}) \simeq 7.50 \pm 0.05$ using Padova isochrones. While the slopes of the resulting MFs based on either metallicity are similar (see Fig. 5, top), the calibration of the solar-metallicity MFs would shift to higher masses.

NGC 1818 has been the subject of several studies aimed at determining its metallicity. Although Johnson et al. (2001) obtained solar metallicity from HST CMD analysis, most other modern (predominantly spectroscopic) determinations centre on either $[\mathrm{Fe} / \mathrm{H}]=-0.37 \pm 0.03$ (e.g., Jasniewicz \& Thévenin 1994; Bonatto et al. 1995) or $[\mathrm{Fe} / \mathrm{H}] \sim-0.8$, roughly corresponding to $Z=0.003$ (e.g., Meliani et al. 1994; Will et al. 1995; Oliva \& Origlia 1998). The MF slopes based on a metallicity of $Z=0.008$ are steeper than for $Z=0.004$ (the lowest metallicity isochrone available for the cluster's young age ${ }^{4}$ ) (see Fig. 5, bottom), although they are both in good agreement with the Kroupa (2001) IMF slope $(\Gamma=0.3 \pm 0.5)$.

The most recent metallicity estimates for NGC 1831 converge to $[\mathrm{Fe} / \mathrm{H}] \sim-0.35$ (Bonatto et al. 1995, based on UV spectroscopy), while Vallenari et al. (1992) similarly suggested a best estimate of $Z=0.008$ based on their analysis of the literature on this cluster at the time of their publication, but see Mateo et al. (1987) and Olszewski et al. (1988, 1991) for close-to-solar abundance estimates. Given the current observational status for this cluster, we adopted $Z=0.008$ as a compromise.

Both NGC 1868 and Hodge 14 are somewhat more metalpoor than the younger clusters in our sample. For NGC 1868, Bica et al. (1986) reported $[\mathrm{Fe} / \mathrm{H}]=-0.6 \pm 0.35$, consistent with Olszewski et al.'s (1991) spectroscopic metallicity determination, $[\mathrm{Fe} / \mathrm{H}]=-0.50$. Similarly, Jensen et al. (1988) and Olszewski et al. (1991) used spectroscopy of Hodge 14 to obtain $[\mathrm{Fe} / \mathrm{H}]=-0.66 \pm 0.2$. For both clusters we adopted $Z=0.004$, corresponding to $[\mathrm{Fe} / \mathrm{H}]=-0.68$.

Finally, NGC 2209 is the lowest-metallicity cluster in our sample, with $[\mathrm{Fe} / \mathrm{H}]=-0.9 \pm 0.3$ (Bica et al. 1986; see also Chiosi et al. 1986; Dottori et al. 1987; Frogel et al. 1990). Based on a careful analysis of the goodness-of-fit parameters for the range of metallicities provides by the Padova isochrones, we adopted $Z=0.004([\mathrm{Fe} / \mathrm{H}]=-0.68)$ for NGC 2209, because its CMD is much more poorly approximated for $[\mathrm{Fe} / \mathrm{H}]=-0.9$.

Metallicity does not play an important role in our analysis of the MF slopes. The models of Baraffe et al. $(1997,1998)$ for different metallicities yield similar main sequences at low mass.

4 Although the Padova models include isochrones for $Z=0.001$, they are only provided for ages in excess of $\log (t / \mathrm{yr})=7.80$. Use of the $Z=0.004$ isochrone results in a cluster age of $\log (t / \mathrm{yr})=7.65 \pm 0.10$. For the low-mass PMS stars, we use $Z=0.003$ and $\log (t / \mathrm{yr})=7.25$ based on the Baraffe et al. $(1997,1998)$ models. 

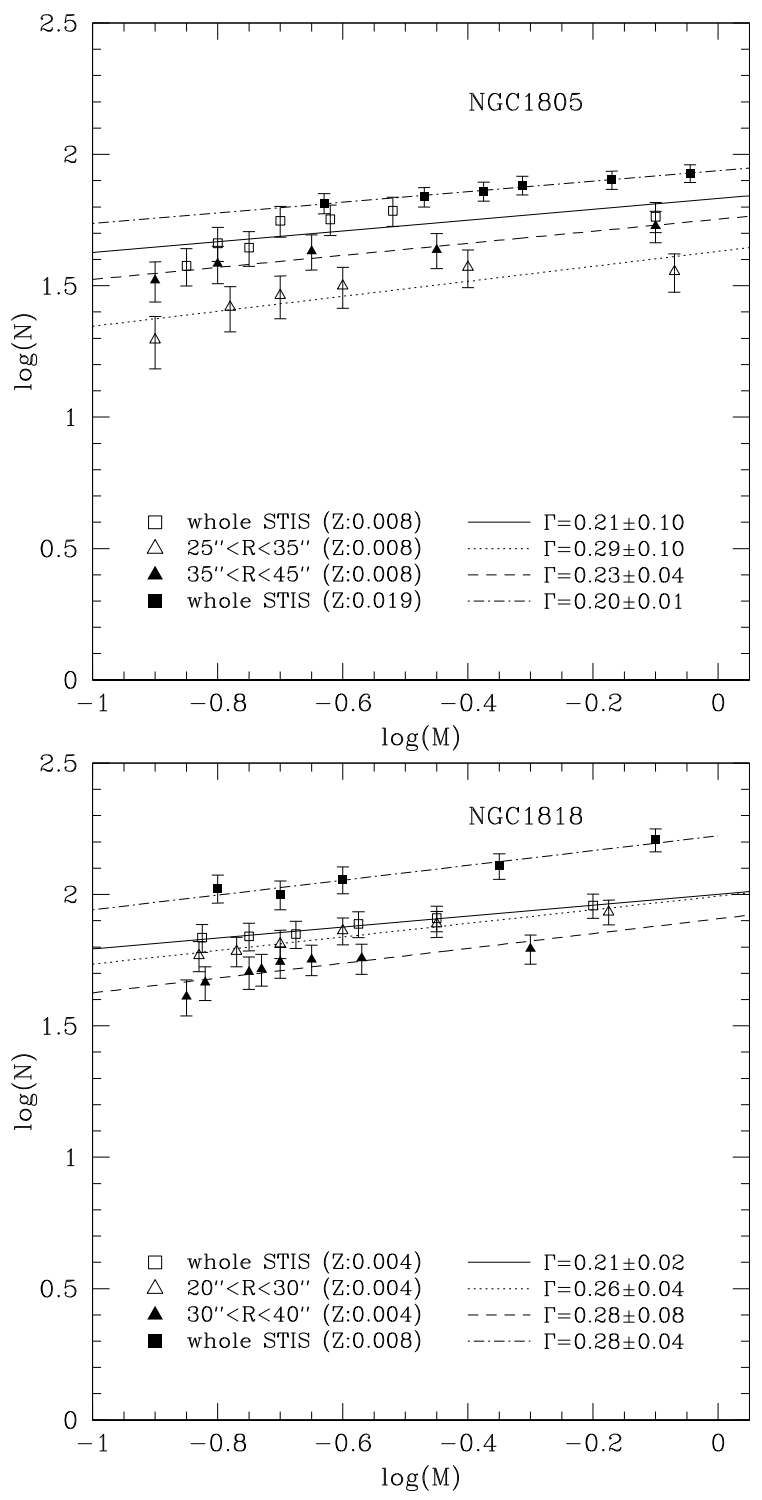

Fig. 5. Low-mass cluster MFs for Pair I, NGC 1805 and NGC 1818.

\subsection{Mass functions}

We adopted the same method as Liu et al. (2009) to derive the MFs of the clusters of pair I. Although the age of NGC 1805 and NGC 1818 is $\log (t / y r) \simeq 7.65$, many low-mass stars are still on the PMS. The ages of the PMS stars in both clusters are about $\log (t / \mathrm{yr}) \simeq 7.25 \pm 0.40$ (Liu et al. 2009). All stars in the clusters of pairs II and III have already evolved onto the main sequence.

Several studies explored the effects of mass segregation in these clusters (based on the same WFPC2 observations used here) by dividing the full field of view into a number of smaller areas at a range of distances from the cluster centres (e.g., Santiago et al. 2001; de Grijs et al. 2002a-c; Kerber et al. 2006). However, our STIS observations were taken at the half-mass radii of our sample clusters and the STIS field is much smaller than that of the combined set of WFPC2 observations, so we limited our analysis to both the entire STIS region and areas at two different radii. Figures 5-7 show the cluster MFs of (and best fits to) different pairs for stellar masses below $1.0 M_{\odot}$, both for the full STIS field and for areas limited by radial range. The results were corrected for sample incompleteness and background contamination. In Liu et al. (2009) we combined the NGC 1818 MFs
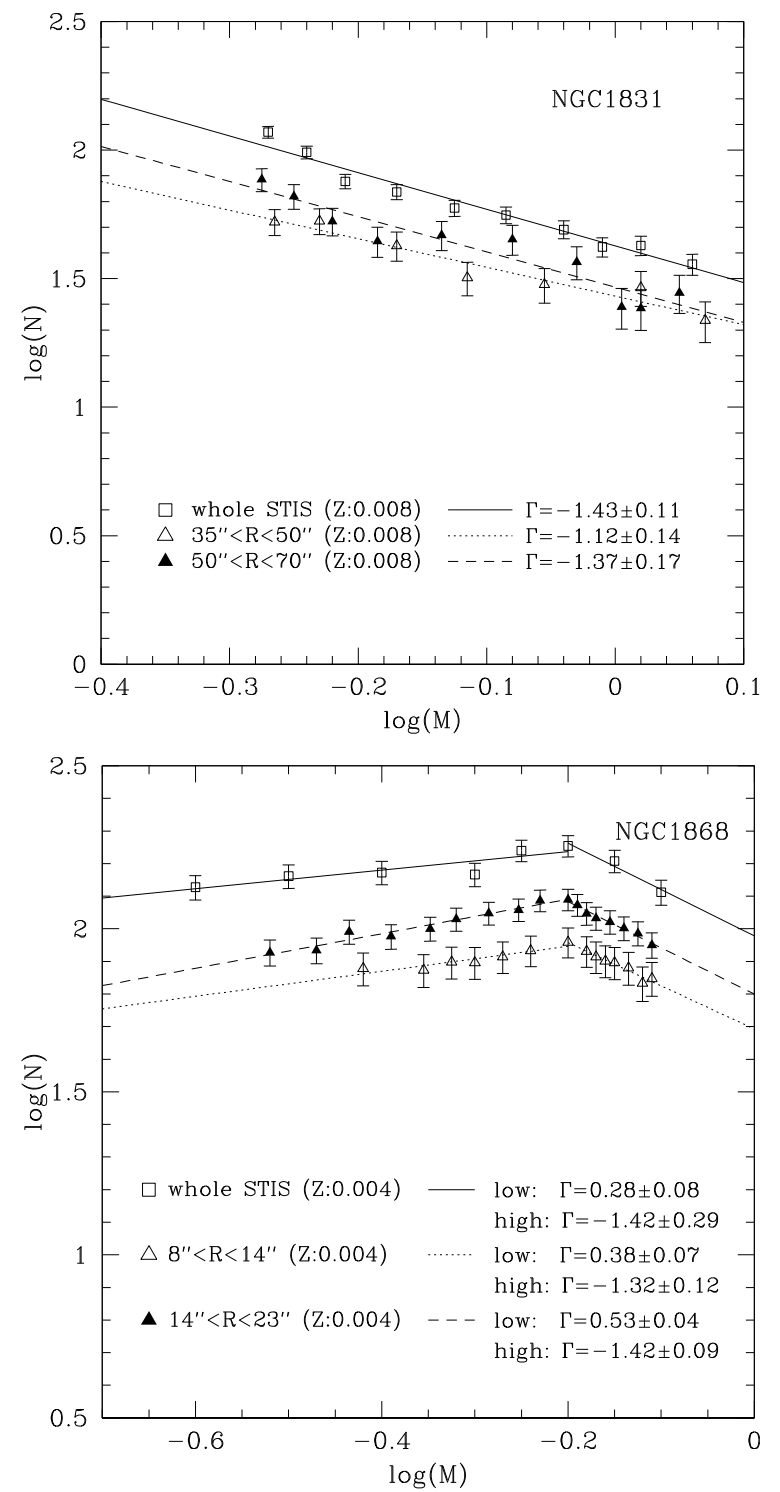

Fig. 6. Low-mass cluster MFs for Pair II, NGC 1831 and NGC 1868.

from WFPC2 and STIS. Using WFPC2, we detected more stars than on the basis of our STIS data for some mass ranges, so the slope of the MF in Liu et al. (2009), $\Gamma=0.46 \pm 0.10$, is steeper than the slope obtained here $(\Gamma=0.28 \pm 0.04)$. However, they both agree with that of the Kroupa (2001) IMF, $\Gamma=0.3 \pm 0.5$, within the uncertainties.

The MFs of NGC 1805, NGC 1818, and NGC 1868 show the same trend, as do the MFs of NGC 1831, NGC 2209, and Hodge 14. Figure 6 shows the change in trend most clearly for our intermediate-age cluster pair. Because NGC 1868 is much more compact than NGC 1831, its dynamical age is much older. This implies that it will be much more evolved dynamically than NGC 1831, resulting in a turnover at much lower masses (outside of our observational range). Similarly, NGC 2209 and Hodge 14 are an order of magnitude older (from both a stellar evolution and a dynamical point of view) than the Pair II clusters, exhibiting declining MFs in the mass range of interest here (see Fig. 7).

We obtained all cluster MFs based only on single stars, neglecting unresolved binary and other multiple stars. As discussed in Liu et al. (2009), Kerber et al. (2006) analysed the effect of binarity on cluster MFs. They found that MFs with binary fractions 

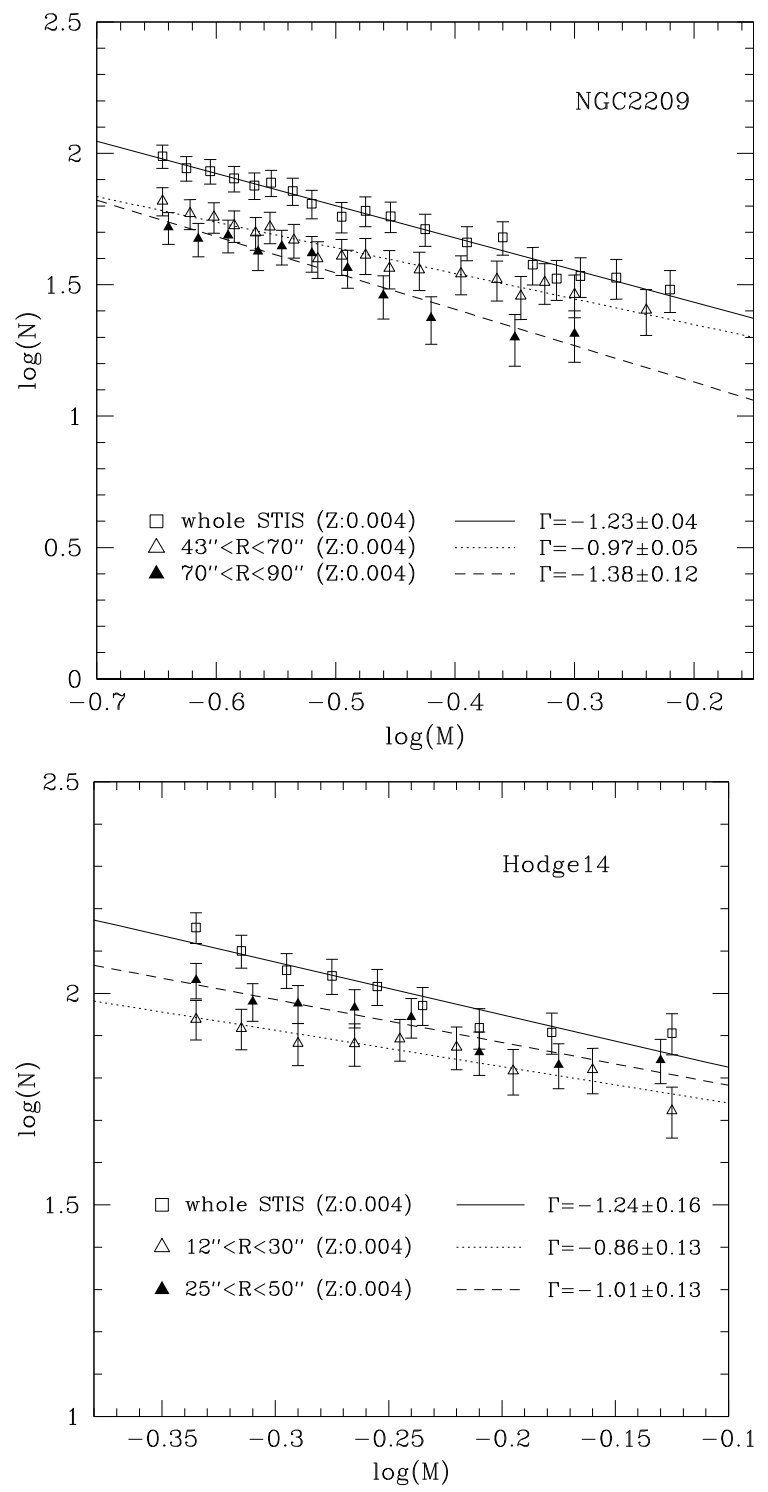

Fig. 7. Low-mass cluster MFs for Pair III, NGC 2209 and Hodge 14.

of unity and $0 \%$ are identical within the observational uncertainties (cf. Liu et al. 2009 for NGC 1818), so that we can justifiably ignore the effects of binarity in the context of the low-mass system MFs derived in this paper (and in Liu et al. 2009).

\section{Discussion}

Owing to both the large distances involved and observational limitations, it is difficult to obtain deep stellar MFs in extragalactic environments. Kroupa (2001) studied the solarneighbourhood IMF down to $\sim 0.01 M_{\odot}$ and reported his oftenquoted broken power-law distribution. Paresce et al. (2000) analysed the MFs of a dozen Galactic GCs down to $0.1 M_{\odot}$ and derived a lognormal distribution below $1.0 M_{\odot}$. Much effort has also focussed on studying stellar MFs in the LMC (e.g., Will et al. 1995; de Grijs et al. 2002b; Gouliermis et al. 2006a,b; Kerber et al. 2006; Da Rio et al. 2009). Recently, the LH 95 IMF obtained by Da Rio et al. (2009) reached down to $0.31 M_{\odot}$, i.e., much deeper than achieved previously beyond the Milky Way. Chiosi et al. (2007) studied the MFs of young SMC clusters down to $0.7 M_{\odot}$. In Liu et al. (2009), we probed - for the first time - the stellar MF in an extragalactic, low-metallicity environment down to $0.15 M_{\odot}$. In this paper, we applied the method of Liu et al. (2009) to all of our sample clusters, aimed at assessing the evolution (if any) of the low-mass MF.

Santiago et al. (2001), de Grijs et al. (2002c), and Kerber et al. (2006) studied the stellar MFs above $1.0 M_{\odot}$ at different radii in these same clusters. They find that the slopes at different radii were significantly different, because significant degrees of mass segregation affect the PDMF shape. de Grijs et al. (2002b) studied the NGC 1805 and NGC $1818 \mathrm{MFs}$ above $1.0 M_{\odot}$. They find that the cluster MFs followed the Salpeter (1955) IMF quite closely. de Grijs et al. (2002c) studied the LFs of all sample clusters and concluded that the PDMFs of the clusters in each pair must be very similar.

The relaxation time at the half-mass radius of compact star clusters can be written as (Meylan 1987)

$t_{\mathrm{r}, \mathrm{h}}=\left(8.92 \times 10^{5}\right) \frac{M_{\mathrm{tot}}^{1 / 2}}{\langle m\rangle} \frac{R_{\mathrm{h}}^{3 / 2}}{\log \left(0.4 M_{\mathrm{tot}} /\langle m\rangle\right)} \mathrm{yr}$,

where $R_{\mathrm{h}}$ is the half-mass radius (in pc), $M_{\mathrm{tot}}$ the total cluster mass (in $M_{\odot}$ ), and $\langle m\rangle$ the typical mass of a cluster star (in $M_{\odot}$ ). The timescale on which a cluster will have lost all traces of its initial conditions is represented well by its half-mass relaxation time. The dynamical properties of the Pair I clusters were discussed by de Grijs et al. (2002b). They (see also de Grijs et al. 2003) computed the half-mass relaxation time as a function of mass for NGC 1805 and NGC 1818. For stellar masses below $1.0 M_{\odot}$, these were $>300$ and $>700 \mathrm{Myr}$, respectively. This is much longer than the clusters' ages, which implies that dynamical cluster evolution will not (or only negligibly) affect the MFs below $1.0 M_{\odot}$ at the clusters' half-mass radii.

Based on the structural parameters of all clusters in Table 1 and their half-mass radii based on the WFPC2 and STIS observations, we calculated the relaxation times of the other clusters in pairs II and III for stellar masses below $1.0 M_{\odot}$. These timescales are $>2.0,0.4,3.9$, and 0.52 Gyr for NGC 1831, NGC 1868, NGC 2209, and Hodge 14, respectively, although dynamical evolution in the cluster core may proceed much faster. Elson et al. (1987) calculated the relaxation time in the core and at the half-mass radius of NGC 1818 as $\log \left(t_{\mathrm{r}}(0) / \mathrm{yr}\right)=$ $8.2-8.8$ and $\log \left(t_{\mathrm{r}, \mathrm{h}} / \mathrm{yr}\right)=9.0-9.7$ (where the age range signifies the uncertainties due to the uncertain mass-to-light ratio). For NGC 1831 the equivalent timescales were found to be $\log \left(t_{\mathrm{r}}(0) / \mathrm{yr}\right)=9.1-9.5$ and $\log \left(t_{\mathrm{r}, \mathrm{h}} / \mathrm{yr}\right)=9.6-10.0$. Compared to the clusters' ages (see Table 1), this implies that both clusters have undergone little or no significant dynamical evolution overall.

From Figs. 5-7 we conclude that the MF slopes for the entire STIS field of view and for smaller areas at different radii are identical within the uncertainties for a given cluster. There may be two reasons for this behaviour: (i) dynamical cluster evolution does not affect the MFs severely below $1.0 M_{\odot}$ beyond the core region, although the relaxation times are shorter than their ages, at least for some clusters; or (ii) the STIS field is fairly small, which prevents us from detecting any differences in the MFs below 1.0 $M_{\odot}$ beyond the crowded centres.

Because of the uniformity of the MFs derived from the full STIS field and from smaller areas at different radii, we compare the MFs of the clusters in each pair in Fig. 8 using the full STIS fields only ${ }^{5}$. In Liu et al. (2009), we adopted both

\footnotetext{
5 Note that the gap in stellar masses for NGC 2209 between the STIS and WFPC2-based MFs is caused by incompleteness differences between both sets of observations, and does not necessarily reflect a true lack of intermediate-mass stars in the cluster.
} 

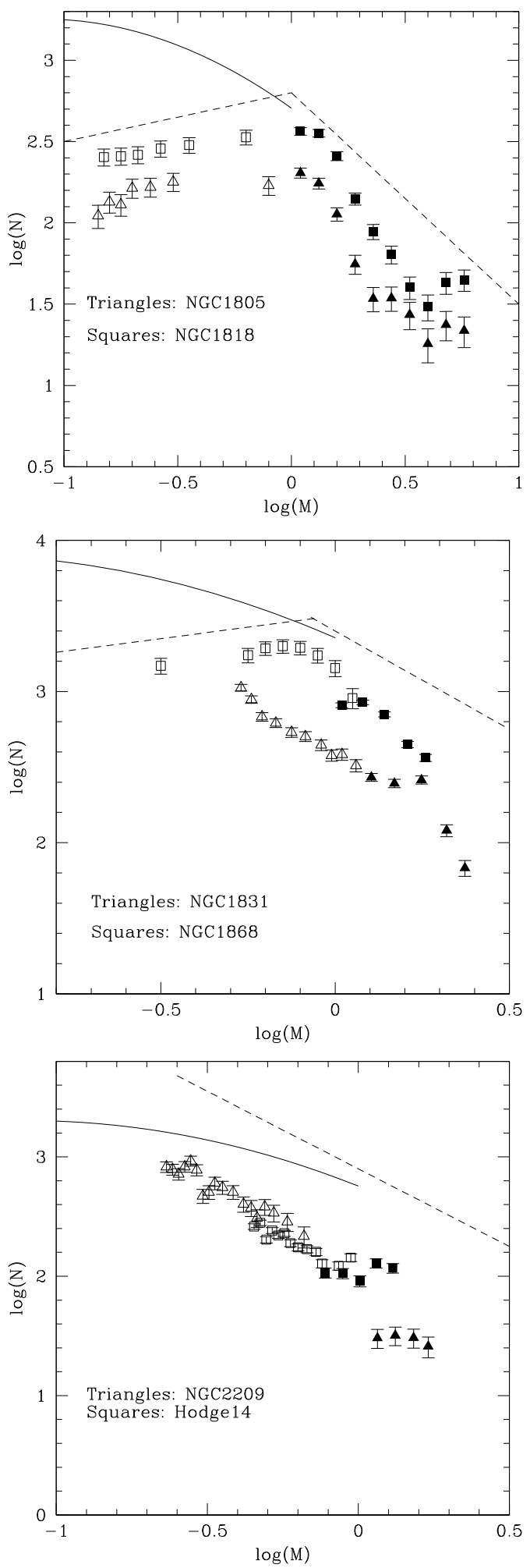

Fig. 8. Full MFs of the sample clusters arranged by pair. All open points are from this paper, based on STIS observations, while the solid points are from de Grijs et al. (2002c), based on WFPC2 observations. The dashed line represents a broken power-law IMF, adopting the Kroupa (2001) high- and low-mass slopes, and the solid curve shows the original Chabrier (2003) lognormal distribution, defined below $1.0 M_{\odot}$, but offset from the data points for reasons of clarity.

broken power-law and lognormal distributions to fit the NGC 1818 MFs. Recent work supports this method (e.g., Covey et al. 2008; Oliveira et al. 2009). In this paper, however, it has
Table 2. Mass functions.

\begin{tabular}{|c|c|c|c|c|}
\hline Cluster & $\log \left(m_{*} / M_{\odot}\right)$ & $N$ & $\log \left(m_{*} / M_{\odot}\right)$ & $N$ \\
\hline \multirow{9}{*}{ NGC 1805} & -0.85 & 110 & 0.20 & 113 \\
\hline & -0.80 & 135 & 0.28 & 56 \\
\hline & -0.75 & 129 & 0.36 & 34 \\
\hline & -0.70 & 164 & 0.44 & 34 \\
\hline & -0.62 & 166 & 0.52 & 27 \\
\hline & -0.52 & 179 & 0.60 & 18 \\
\hline & -0.10 & 170 & 0.68 & 24 \\
\hline & 0.04 & 203 & 0.76 & 22 \\
\hline & 0.12 & 175 & & \\
\hline \multirow[t]{8}{*}{ NGC 1818} & -0.825 & 254 & 0.20 & 258 \\
\hline & -0.75 & 257 & 0.28 & 141 \\
\hline & -0.675 & 262 & 0.36 & 88 \\
\hline & -0.575 & 286 & 0.44 & 64 \\
\hline & -0.45 & 301 & 0.52 & 40 \\
\hline & -0.20 & 336 & 0.60 & 30 \\
\hline & 0.04 & 366 & 0.68 & 43 \\
\hline & 0.12 & 357 & 0.76 & 45 \\
\hline \multirow[t]{8}{*}{ NGC 1831} & -0.27 & 1059 & 0.02 & 383 \\
\hline & -0.24 & 884 & 0.06 & 324 \\
\hline & -0.21 & 681 & 0.104 & 271 \\
\hline & -0.17 & 620 & 0.17 & 247 \\
\hline & -0.125 & 536 & 0.248 & 260 \\
\hline & -0.085 & 504 & 0.32 & 121 \\
\hline & -0.04 & 442 & 0.372 & 68 \\
\hline & -0.01 & 378 & & \\
\hline \multirow[t]{6}{*}{ NGC 1868} & -0.50 & 1479 & 0.00 & 1429 \\
\hline & -0.25 & 1738 & 0.05 & 906 \\
\hline & -0.20 & 1932 & 0.08 & 847 \\
\hline & -0.15 & 1995 & 0.14 & 706 \\
\hline & -0.10 & 1945 & 0.21 & 447 \\
\hline & -0.05 & 1734 & 0.26 & 364 \\
\hline \multirow[t]{11}{*}{ NGC 2209} & -0.635 & 825 & -0.38 & 403 \\
\hline & -0.615 & 786 & -0.355 & 374 \\
\hline & -0.595 & 718 & -0.335 & 309 \\
\hline & -0.575 & 828 & -0.31 & 381 \\
\hline & -0.555 & 927 & -0.28 & 340 \\
\hline & -0.535 & 777 & -0.235 & 287 \\
\hline & -0.515 & 471 & -0.18 & 216 \\
\hline & -0.495 & 508 & 0.063 & 30 \\
\hline & -0.475 & 603 & 0.122 & 32 \\
\hline & -0.45 & 555 & 0.183 & 31 \\
\hline & -0.415 & 508 & 0.23 & 26 \\
\hline \multirow[t]{8}{*}{ Hodge 14} & -0.345 & 262 & -0.17 & 169 \\
\hline & -0.325 & 281 & -0.14 & 160 \\
\hline & -0.305 & 203 & -0.12 & 128 \\
\hline & -0.285 & 242 & -0.065 & 122 \\
\hline & -0.265 & 220 & -0.025 & 143 \\
\hline & -0.245 & 229 & 0.005 & 91 \\
\hline & -0.225 & 190 & 0.059 & 129 \\
\hline & -0.20 & 175 & 0.114 & 117 \\
\hline
\end{tabular}

become clear that not all cluster MFs below $1.0 M_{\odot}$ show an obvious turnover. Therefore, we adopted power laws to fit all cluster MFs below 1.0 $M_{\odot}$. We include the relevant parameters, including those for the standard Kroupa (2001) IMF, in Table 3. The MF of NGC 1805 shows the same result as for NGC 1818 in Liu et al. (2009); i.e., their slopes are identical to the Kroupa (2001) IMF within the uncertainties, although the applicable mass ranges extend to higher masses than the relevant Kroupa (2001) slope (likely due to statistical fluctuations; cf. Liu et al. 2009). NGC 1868 exhibits a broken power-law distribution similar to the Kroupa (2001) IMF and the complete IMFs of NGC 1805 and NGC 1818 (de Grijs et al. 2002b; Liu et al. 2009). The MF of NGC 1831 does not show a turnover, but its 
Table 3. Mass function slopes.

\begin{tabular}{ccccc}
\hline \hline \multirow{2}{*}{ Sample } & \multicolumn{2}{c}{ Low mass } & \multicolumn{2}{c}{ High mass } \\
& Slope $(\Gamma)$ & Mass $\left(M_{\odot}\right)$ & Slope $(\Gamma)$ & Mass $\left(M_{\odot}\right)$ \\
\hline Kroupa & $0.3 \pm 0.5$ & $0.08-0.5$ & $-1.3 \pm 0.3$ & $0.5-1.0$ \\
$(2001)$ & & & $-1.3 \pm 0.7$ & $\geq 1.0$ \\
NGC 1805 & $0.21 \pm 0.10$ & $0.14-0.79$ & & \\
NGC 1818 & $0.21 \pm 0.02$ & $0.15-0.63$ & & \\
NGC 1831 & & $-1.43 \pm 0.11$ & $0.54-1.15$ \\
NGC 1868 & $0.28 \pm 0.08$ & $0.25-0.63$ & $-1.42 \pm 0.29$ & $0.63-0.79$ \\
NGC 2209 & & $-1.23 \pm 0.04$ & $0.23-0.60$ \\
Hodge14 & & $-1.24 \pm 0.16$ & $0.46-0.75$ \\
\hline
\end{tabular}

slope in the mass range from 0.54 to $1.15 M_{\odot}$ is identical to that of the Kroupa (2001) IMF for masses between 0.5 and $1.0 M_{\odot}$, again within the uncertainties. The MF slopes of NGC 2209 and Hodge 14 are also identical to those of the Kroupa (2001) IMF below $1.0 M_{\odot}$.

In this paper, we extended our previous work (Liu et al. 2009) to all LMC sample clusters. The MFs of the clusters in each pair exhibit identical slopes, and they are all also identical to the standard Kroupa (2001) IMF below 1.0 $M_{\odot}$, independent of metallicity, particularly for the clusters in pair I (de Grijs et al. 2002b; Liu et al. 2009) and NGC 1868 in pair II (this paper). de Grijs et al. (2002c) studied the LFs of all sample clusters above $1.0 M_{\odot}$. We converted the LFs to MFs based on the Padova isochrones for the appropriate metallicities and combine both of our results to obtain complete PDMFs for all sample clusters (see Fig. 8 and Table 2). All MFs are similar to the standard Kroupa (2001) IMF, and the MFs of NGC 1831, NGC 2209, and Hodge 14 also match the Chabrier (2003) lognormal distribution, at least qualitatively although not in detail (as shown by the solid lines in the individual panels).

\section{Summary and conclusions}

We extended our pilot study in Liu et al. (2009) and used deep HST/STIS photometry of a carefully selected sample of rich, compact clusters in the LMC to derive their stellar MFs for masses below $1.0 M_{\odot}$, which we combined with the MFs of de Grijs et al. (2002c) above $1.0 M_{\odot}$ to obtain complete MFs for all sample clusters. To our knowledge, this is the first time, along with Liu et al. (2009), that anyone has probed stellar (cluster) MFs to this depth in an extragalactic, low-metallicity environment. Based on our STIS observations, the MFs of our compact clusters are all identical to the standard Kroupa (2001) IMF below $1.0 M_{\odot}$, within the uncertainties.

The observations were taken beyond the crowded cluster cores and only included stars at the half-mass radii. In addition, the relaxation timescales of low-mass stars are much longer than the equivalent periods for high-mass stars, so that dynamical evolution will not have affected the younger clusters' stellar mass distributions below $1.0 M_{\odot}$ noticeably. We have therefore provided unprecedented insights into the IMF in a low-density and low-metallicity extragalactic environment.

Acknowledgements. This work was supported by the National Natural Science Foundation of China under grant No. 10573022 and by the Ministry of Science and Technology of China under grant No. 2007CB815406. RdG acknowledges partial financial support from the Royal Society in the form of a UK-China International Joint Project. We thank Isabelle Baraffe for advice and discussions in earlier stages of this project. This paper is based on archival observations with the NASA/ESA Hubble Space Telescope, obtained at the Space Telescope Science Institute, which is operated by the Association of Universities for Research in Astronomy, Inc., under NASA contract NAS 5-26555. This research has made use of NASA's Astrophysics Data System Abstract Service.

\section{References}

Andersen, M., Meyer, M. R., Greiss1, J., \& Aversa, A. 2008, ApJ, 683, L183 Baraffe, I., Chabrier, G., Allard, F., \& Hauschildt, P. H. 1997, A\&A, 327, 1054 Baraffe, I., Chabrier, G., Allard, F., \& Hauschildt, P. H. 1998, A\&A, 337, 403 Beaulieu, S. F, Elson, R. A. W., Gilmore, G. F., et al. 1999, in: New Views of the Magellanic Clouds, ed. Y.-H. Chu, N. Suntzeff, J. Hesser, \& D. Bohlender (ASP: San Francisco), IAU Symp., 190, 460

Bica, E., Dottori, H., \& Pastoriza, M. 1986, A\&A, 156, 261

Bica, E., Clariá, J. J., Dottori, H., Santos, J. F. C., \& Piatti, A. E. 1996, ApJS, 102,57

Bonatto, C., Bica, E., \& Alloin, D. 1995, A\&AS, 112, 71

Castro, R., Santiago, B. X., Gilmore, G. F., Beaulieu, S., \& Johnson, R. A. 2001, MNRAS, 326, 333

Chabrier, G. 2003, PASP, 115, 763

Chiosi, E., \& Vallenari, A. 2007, A\&A, 466, 165

Chiosi, C., Bertelli, G., Bressan, A., \& Nasi, E. 1986, A\&A, 165, 84

Covey, K. R., Hawley, S. L., Bochanski, J. J., et al. 2008, AJ, 136, 1778

Da Rio, N., Gouliermis, D., \& Henning, T. 2009, ApJ, 696, 528

de Grijs, R., Johnson, R. A., Gilmore, G. F., \& Frayn, C. M. 2002a, MNRAS, 331,228

de Grijs, R., Gilmore, G. F., Johnson, R. A., \& Mackey, A. D. 2002b, MNRAS, 331,245

de Grijs, R., Gilmore, G. F., Mackey, A. D., et al. 2002c, MNRAS, 337, 597

Gilmore, G. F., \& Johnson, R. A. 2003, in: The Local Group as an Astrophysical Laboratory, STScI Symp., ed. M. Livio, \& T. M. Brown, STScI: Baltimore, 20 [arXiv: astro-ph/0305262]

Dottori, H., Melnick, J., \& Bica, E. 1987, RMxAA, 14, 183

Elson, R. A. W., Fall, S. M., \& Freeman, K. C. 1987, ApJ, 323, 54

Elson, R., Sigurdsson, S., Davies, M., Hurley, J., \& Gilmore, G. 1998, MNRAS, 300,857

Elson, R., Tanvir, N., Gilmore, G., Johnson, R. A., \& Beaulieu, S. 1999, in: New Views of the Magellanic Clouds, ed. Y.-H. Chu, N. Suntzeff, J. Hesser, \& D. Bohlender (ASP: San Francisco), IAU Symp., 190, 417

Frogel, J. A., Mould, J., \& Blanco, V. M. 1990, ApJ, 352, 96

Girardi, L., Bressan, A., Bertelli, G., \& Chiosi, C. 2000, A\&AS, 141, 371

Gouliermis, D., Brandner, W., \& Henning, Th. 2006a, ApJ, 641, 838

Gouliermis, D., Brandner, W., \& Henning, Th. 2006b, ApJ, 636, L133

Holtzman, J. A., Burrows, C. J., Casertano, S., et al. 1995, PASP, 107, 1065

Jasniewicz, G., \& Thévenin, F. 1994, A\&A, 282, 717

Jensen, J., Mould, J. R., \& Reid, I. N. 1988, ApJS, 67, 77

Johnson, R. A., Beaulieu, S. F., Gilmore, G. F., et al. 2001, MNRAS, 324, 367

Kerber, L. O., \& Santiago, B. X. 2006, A\&A, 452, 155

Krist, J., \& Hook, R. 2001, The Tiny Tim User's Guide, Version 6.0

(http://www.stsci.edu/software/tinytim/)

Kroupa, P. 2001, MNRAS, 322, 231

Liu, Q., de Grijs, R., Deng, L. C., et al. 2009, MNRAS, in press [arXiv: 0903.4787]

Mackey, A. D., \& Gilmore, G. F. 2003, MNRAS, 338, 85

Mateo, M., \& Hodge, P. 1987, ApJ, 320, 626

Meliani, M. T., Barbuy, B., \& Richtler, T. 1994, A\&A, 290, 753

Meurer, G. R., Cacciari, C., \& Freeman, K. C. 1990, AJ, 99, 1124

Meylan, G. 1987, A\&A, 184, 144

Oliva, E., \& Origlia, L. 1998, A\&A, 332, 46

Oliveira, J. M., Jeffries, R. D., \& van Loon, J. T. 2009, MNRAS, 392, 1034

Olszewski, E. W., Harris, H. C., Schommer, R. A., \& Canterna, R. W. 1988, AJ, 95,84

Olszewski, E. W., Schommer, R. A., Suntzeff, N. B., \& Harris, H. C. 1991, AJ, 101,515

Paresce, F., \& De Marchi, G. 2000, ApJ, 534, 870

Park, B., Sung, H., Bessell, M., \& Kang, Y. 2000, AJ, 120, 894

Salpeter, E. E. 1955, AJ, 101, 1865

Santiago, B. X., Beaulieu, S., Johnson, R., \& Gilmore, G. F. 2001, A\&A, 369, 74

Vallenari, A., Chiosi, C., Bertelli, G., Meylan, G., \& Ortolani, S. 1992, AJ, 104, 1100

Westerlund, B. E. 1990, A\&AR, 2, 29

White, R. J., Ghez, A. M., Reid, I. N., \& Schultz, G. 1999, ApJ, 520, 811

Whitmore, B., Heyer, I., \& Casertano, S. 1999, PASP, 111, 1559

Will, J. M., Bomans, D. J., \& de Boer, K. S. 1995, A\&A, 295, 54 